DOI: 10.12957/demetra.2016.16103

\title{
Prácticas alimentarias de personas con ERG desde la perspectiva de quienes la padecen y sus cuidadores. Un estudio cualitativo en México
}

\author{
Food practices of people with CKD from the perspective of sufferers and their caregivers. \\ A qualitative study in Mexico
}

\author{
Julieta Nataly Peralta Suchil ${ }^{1}$ \\ Luis Eduardo Hernandez Ibarra' \\ 1 Universidad Autónoma de San Luis Potosí, \\ UASLP. San Luis Potosí, S.L.P., México. \\ Correspondencia / Correspondence \\ Luis Eduardo Hernández Ibarra \\ E-mail: eduardo_ibarra@hotmail.com
}

\section{Resumen}

Objetivo: Conocer las prácticas alimentarias de personas que padecen la enfermedad renal crónica desde la perspectiva de quienes la padecen y sus cuidadores. Metodología: Se llevó a cabo un estudio cualitativo con acercamiento etnográfico en San Luis Potosí, México. Se formaron grupos focales, con los cuales se realizaron entrevistas semiestructuradas a 16 personas con enfermedad renal crónica y a 22 de sus cuidadores. Se realizó el análisis del contenido temático. Resultados: Los participantes del estudio señalaron haber incorporado recomendaciones con relación a la ingesta de sal, grasa y azúcar, así como a la cantidad de agua que beben. Sin embargo, adaptar el régimen prescrito a la dieta familiar y comer fuera de casa son las principales dificultades, sobre todo en los días en que acuden a las terapias sustitutivas o a consulta con el médico. El uso y consumo de remedios caseros o tradicionales es una práctica común entre los participantes del estudio, debido a la esperanza de mejorar su condición de salud. Conclusiones: Las prácticas alimentarias de las personas con ERC que participaron en el estudio no coinciden con las recomendaciones dietéticas prescritas para el tratamiento de su enfermedad. Eso puede asociarse principalmente a dificultades económicas, creencias y costumbres. Es necesario realizar más estudios de corte cualitativo y participativo que incorporen las experiencias y prácticas de los propios enfermos y sus cuidadores para comprender mejor las situaciones que interfieren en una adecuada alimentación de quienes padecen enfermedad renal crónica.

Palabras clave: Insuficiencia renal crónica. Dieta. Investigación Cualitativa. Hábitos Alimenticios. 


\section{Abstract}

Objective: This study aims to determine the dietary practices of people suffering from chronic kidney disease from the perspective of sufferers and their caregivers. Methodology: A qualitative study was conducted with ethnographic approach in San Luis Potosi, Mexico. Focus groups and semi-structured interviews with 16 people with chronic kidney disease and 22 caregivers were performed. The thematic content analysis was performed. Results: The study participants reported having incorporated recommendations regarding the intake of salt, fat and sugar, as well as the amount of drinking water. However, adapting to the family diet prescribed regime and eating out are the main difficulties, especially attending the day of replacement therapies and medical consultations. The use and consumption of homemade or traditional remedies is a common practice among study participants, hoping to improve their health condition. Conclusions: The dietary practices of people with CKD who participated in the study did not match the prescribed dietary recommendations for the treatment of their disease. This may be associated mainly to economic difficulties, beliefs and customs. Further qualitative and participative studies that incorporate practices of the sufferers and their caregivers are needed to better understand the situations that interfere with the ingestion of an adequate diet of those suffering from chronic kidney disease.

Key words: Renal Insufficiency Chronic. Diet. Qualitative Research. Food Habits.

\section{Introducción}

La Enfermedad Renal Crónica (ERC) es uno de los principales problemas de la salud pública en el mundo. Y México ocupa el segundo lugar en incidencia de ERC, con aproximadamente 425 casos por un millón de habitantes. ${ }^{1,2}$ Ella ha generado una gran demanda de recursos humanos, económicos y de infraestructura que involucran tanto los sistemas de salud, como a las personas que la padecen y sus familias.

La enfermedad renal es una enfermedad compleja, que requiere un tratamiento multidisciplinario e integral. Dicho tratamiento consta de tres pilares fundamentales: la terapia sustitutiva, la terapia farmacológica y un régimen alimenticio adecuado. Muchas veces, se olvida el pilar alimentario o se lo deja a un lado, a pesar de su importancia en el tratamiento de la enfermedad renal. Aunque la prescripción de la dieta varíe según el estadío de la enfermedad, 
su objetivo es evitar la progresión del daño renal, reponer las pérdidas nutricionales y evitar las complicaciones metabólicas y la desnutrición, consecuencias frecuentes en las personas que padecen la ERC.

Se ha documentado que seguir el régimen dietético para el manejo de la enfermedad renal no es una tarea fácil, porque requiere cambios en el estilo de vida, lo que es poco probable que ocurra cuando la intervención nutricional se basa en un sistema convencional y clínico, o cuando no se tiene acceso al personal de salud que oriente sobre el tema. ${ }^{3}$ Es bien sabido también que, si la persona con ERC no sigue este régimen, puede presentar complicaciones para su salud, como la retención de líquidos, hipertensión, proteinuria, hiperkalemia, hiperfosfatemia, enfermedades óseas e incremento de los niveles de urea y creatinina, alteraciones que agravan su condición clínica y reducen la calidad y esperanza de vida.,

A pesar de que cada vez existen más estudios que exploran los aspectos sociales y culturales de la enfermedad renal (Mercado-Martínez y colaboradores, 2014; Mercado-Martínez \& Urias-Vázquez 2014), poco se ha profundizado en la temática sobre la relación entre la nutrición, alimentación y enfermedad renal crónica. Por todo eso, el presente trabajo examina las experiencias y prácticas de las personas con enfermedad renal crónica que se encuentran en hemodiálisis, así como de sus familiares, en torno al hecho de seguir el régimen dietético prescrito.

\section{Material y Métodos}

Se realizó un estudio cualitativo con acercamiento etnográfico, durante el periodo de enero de 2013 a agosto de 2014, en un municipio ubicado al sur de San Luis Potosí, México. Los informantes fueron seleccionados a partir de un muestreo teórico. ${ }^{6}$ Participaron en el estudio 16 personas con enfermedad renal crónica en tratamiento de hemodiálisis de las cuales nueve eran hombres y siete mujeres, así como 22 familiares que actuaban como cuidadores, 18 mujeres y 4 hombres. (Ver tablas 1 y 2).

Se realizaron entrevistas semiestructuradas a las personas enfermas, mientras que los cuidadores participaron en grupos focales según la propuesta de Morgan. Las entrevistas se llevaron a cabo en los hogares de los participantes, y los grupos focales en la sede de una asociación civil que apoya a personas con dicho padecimiento. También se llevó a cabo la observación en mercados y establecimientos que venden comida en el municipio y lugares cercanos a las unidades de hemodiálisis.

Las entrevistas, grupos focales y notas de campo fueron digitalizados, y los archivos generados fueron guardados y codificados para el uso exclusivo de los investigadores. La transcripción de entrevistas se realizó con la ayuda del Software F4, y posteriormente se las validó para corroborar la calidad y veracidad de la transcripción. 
Se realizó el análisis de contenido temático convencional de acuerdo con la propuesta de Hsieh y Shannon ${ }^{7}$. Los códigos y categorías se obtuvieron directamente de las transcripciones de entrevistas y notas de campo realizadas, teniendo en cuenta las preguntas de la investigación y los objetivos del estudio. Para eso, se realizó una lectura en extenso de las transcripciones, que posteriormente fueron capturadas con la ayuda del programa Etnograph v.6, para iniciar la codificación. Los códigos generados fueron registrados en una matriz para facilitar la identificación de relaciones, organización de temas, creación de familias y categorías de códigos relacionados. Los códigos y categorías emergentes se revisaron continuamente durante el proceso de análisis de forma que el esquema de códigos inicial se fue modificando y, una vez que se obtuvo un esquema final, se otorgó una definición a las categorías.

El presente estudio fue sometido para consideración al Comité de Ética en Investigación de la Facultad de Enfermería (CEIFE), en San Luis Potosí, para lo cual se consideraron los lineamientos de la Ley General de Salud de México ${ }^{8}$ y los estatutos de la Declaración de Helsinki de la Asociación Médica Mundial, para su elaboración. ${ }^{9}$ En función de estas declaraciones se obtuvo el siguiente registro: CEIFE-2013-062.

\section{Resultados}

Los cambios que las personas con ERC deben realizar para adecuar su alimentación al régimen dietético recomendado para su padecimiento difieren completamente de las prácticas alimentarias que solían llevar a cabo. Estas diferencias van desde la restricción o aumento de ciertos alimentos por su contenido en proteínas, potasio, fosforo y sodio, restricción de líquidos, formas de preparación de alimentos, ajuste de horarios y tiempos de comida, hasta la compra de alimentos o suplementos que se consideren necesarios para controlar la evolución del padecimiento. En ese sentido, no solo se ve afectada la persona que padece la enfermedad, sino también su cuidador principal e incluso el resto de la familia.

A continuación se describen los principales hallazgos en torno a las prácticas alimentarias y estrategias que han aplicado al intentar adecuarse al régimen dietético recomendado tanto las personas con ERC como sus cuidadores:

\section{La restricción como eje del tratamiento}

La enfermedad renal crónica, en voz de quienes participaron en este estudio, se vive como una enfermedad íntimamente vinculada a las restricciones alimentarias, específicamente con relación a los líquidos. Por lo tanto, es una constante escucharlos relatar sobre la sensación de hambre y sed permanente debido a que no quedan satisfechos con cantidades que se les recomiendan o 
prescriben. En eso coinciden tanto los hombres como las mujeres con el padecimiento, sean jóvenes o adultos. Uno de ellos lo describe de la siguiente forma

...te restringen todo, leche, carne, huevo, también frutas y verduras que son altas en potasio y fósforo. Todas esas cosas me las empezaron a restringir; entonces tenía muy pocas opciones. Eso es de lo más difícil... la sal y el agua es lo que más duele...

Un aspecto recurrente que surge de los discursos de quienes padecen la enfermedad renal crónica y de sus familiares es la forma cómo describen y se refieren a la dieta prescrita. Quienes participaron en el estudio suelen referirse a "la hoja" o "la hojita" cuando aluden a la prescripción por escrito que les dieron durante la consulta. Eso al referirse al plan estandarizado que les dan después de la consulta y que pareciera ser el único recurso con el que cuenta quien les brinda atención nutricional. Según las personas entrevistadas, tal forma sólo les fue útil al inicio del padecimiento; generalmente la dejan a un lado por lo complicado que les parece o por no entender las indicaciones. Así se refiere a ella uno de los participantes:

Esa hojita que me dieron [formato impreso de la dieta prescrita]; sí la lei pero no le entendí. Haz de cuenta, esta es la hoja y me le ponían puros cuadritos. No, la mera verdad ni les entendía...

A pesar de que la mayoría reconocía la importancia del régimen prescrito y estaba de acuerdo en que las dietas eran necesarias para el manejo y control de su enfermedad, incluso al referirse a la terminología médica y nutricional, coincidía en el hecho de haber seguido la dieta sólo al inicio del tratamiento. Los pacientes también mencionaban que, con el paso del tiempo, iban perdiendo dicho control y llegaban a comer todo tipo de alimentos debido al cansancio y hastío; o decidían no seguir la dieta o no cumplirla al pie de la letra por los costos que les representaba. Otro de los participantes señaló al respecto,

...yo estoy tratando de apegarme lo más que puedo a la dieta. Pero al paso del tiempo, te voy a ser honesto, se llega a perder, se llega a perder y llegas a consumir de todo. Yo estoy consumiendo ahorita básicamente de todo...

Otro cambio que los informantes mencionaron es la disminución en el consumo de agua más que de otros líquidos; la mayoría de ellos continua consumiendo refresco. Quienes han seguido esta restricción comentaron que utilizan vasos y botellas graduadas o de una cantidad específica para lograr consumir la cantidad de líquidos que se les permite. Así lo refirieron algunos de ellos:

...tenemos un vaso donde tiene la medida, le dice su bebé de ella, "no tío, ese es mío" porque le damos en un vaso de bebé, también agarramos la medida en las botellas de agua... Cuidador. Ent.05 
La forma de preparar los alimentos es otra de las prácticas que se cambia cuando hay una persona con ERC en casa. Algunos cuidadores mencionaron que han eliminado el azúcar, la sal y los sazonadores en sus preparaciones, otros solo han reducido la cantidad que se agrega a los alimentos o definitivamente siguen cocinando utilizando indiscriminadamente alguno de estos ingredientes. Los siguientes testimonios dan cuenta de ello:

....ya no uso la sal [H: nada] esa si ni por equivocación eh, nada de sal, todo se lo cocino sin sal... Cuidador. Ent. 07 ...yo cocino normal, porque a él no le gusta la comida sin sal..." Cuidador. Ent. 11

El hecho de cocinar sin sal es uno de los factores que más influye en el sabor de los alimentos y, por lo tanto, en la decisión de la persona con ERC de comerlos o no. Ante esta situación, los cuidadores utilizan sustitutos de la sal, mantequilla, hierbas de olor y especias, para mejorar la palatabilidad. Una cuidadora describe esta práctica:

...no utilizo la sal ni el consomate [sazonador de alimentos], eso no, puras cosas naturales, la ramita de mejorana, la ramita de perejil, la ramita de cilantro es lo que uso para cocinar... Cuidador. Ent. 10

...me decía “no es que a mí no me gusta así [sin sal]”, pues ni modo "pónmele siquiera tantita mantequilla” y eso hago, porque tampoco puedo ponerle sal con ajo, consomé de pollo, nada de eso... Persona con ERC. Ent. 07

Otro cambio que han tenido que adoptar en las preparaciones es el remojar las leguminosas y carnes antes de cocinarlas. Esta práctica ha sido adoptada por recomendación del nutriólogo, los informantes refirieron que este proceso es necesario para eliminar toxinas en los alimentos, las siguientes citas reflejan estos cambios en la preparación de alimentos:

...si le doy bistec de res, lo pongo a remojar media hora en agua fría, igual el filete de pescado, el pollo, lo que sea de carne lo tengo que poner para quitar las toxinas..."Cuidador. Ent. 07

....sustituimos la carne por frijoles, pero también tienen mucho fósforo, entonces procuramos tirarles la primera agua, que es parte de lo que recomiendan los nutriólogos..." Cuidador. Ent.06

\section{Adaptaciones familiares ante el régimen dietético cuando se padece ERC}

Todas las personas con ERC que participaron en este estudio viven con sus familiares, por lo que los cuidadores, que generalmente son la madre o la esposa, se ven ante la necesidad de ajustar la dieta familiar para que atienda a las necesidades de la persona con el padecimiento. Ese proceso es complejo, pues los alimentos y las formas de prepararlos a las que el familiar debe apegarse son muy diferentes a las de la dieta habitual. 
Existen diversas estrategias por medio de las cuales la familia se ajusta a las necesidades alimentarias de quien padece ERC. La mayoría de los cuidadores mencionó que hace platillos por separado, uno para el familiar con el padecimiento y otro para el resto de la familia. Así lo expresaron algunos cuidadores:

...siempre hay que hacerle de comer a ella aparte y la señora que trabaja aquí ya sabe que ella [su hija] está enferma, y le dice, "oye me dijeron que hiciera esto de comer ia ti que te hago?"... Cuidador. Ent. 06

En otras familias, se decide preparar el mismo platillo y solo intercambiar el alimento que no se puede comer por otro que si se pueda incluir en la dieta de quien padece ERC y dejar como opción a cada miembro de la familia el agregar aquellos que no se incluyan.

Los cuidadores coincidieron que con el paso del tiempo asimilar esos cambios se vuelve más fácil y llegan a acostumbrarse a dejar de comer ciertos alimentos. El cambio al que mencionaron estar más acostumbrados es evitar el consumo de la sal. Dos cuidadores hablan de este cambio en su alimentación:

...no pues al paso del tiempo nos fuimos acostumbrando. Igual mis hijos decian: "iay, no tiene sal!". Pues ni modo, asi debe de ser, y lo han aceptado... Cuidador. Ent. 07

Las comidas en familia, en ocasiones, pueden dar lugar a que la persona con ERC se enfrente a situaciones de tentación al encontrar en la mesa aquellos alimentos que se le han restringido.

Los cuidadores comentaron que evitan consumir, llevar a la mesa o comprar los alimentos que su familiar no puede comer, precisamente para evitar las tentaciones. Dos cuidadoras hablan al respecto:

...nosotros optamos en la casa. Porque lo que no pueden comer, ella [su hermana] y yo no lo volvemos a hacer y punto, se acabó, no les damos tentación. Lo que pueden comer ellos es lo que hacemos de comer... Cuidador. Gf. 05

En otros hogares, no se ha hecho ningún ajuste a la dieta familiar, y esta se mantiene exactamente igual para todos los miembros de la familia. Una cuidadora habla sobre la alimentación en su casa:

... aquí en casa, a la hora de la comida, así como comemos nosotros come él. Aunque está enfermo, él come igual que todos... Cuidador. Ent. 10 


\section{Adaptación del régimen dietético en fiestas y eventos sociales}

Las fiestas y eventos sociales son situaciones que amenazan el seguimiento del régimen alimenticio de las personas con ERC, pues por lo general los alimentos que se ofrecen no son recomendables para que ellos los consuman. Los informantes mencionaron que el platillo típico en estas ocasiones es el asado de boda, mole y barbacoa. Esos platos son preparados a base de carne, regularmente de cerdo, sal, azúcar, variedad de chiles, chocolate y especias, acompañados de refresco o cerveza.

Ante tales circunstancias, ellos prefieren evitar comer en esos eventos. Las estrategias que aplican en esos casos son comer antes de acudir a la fiesta, comer cuando regresan a casa o comer solo los alimentos que no les provoquen malestar, como el arroz o tortilla. Algunos informantes describen esta situación:

... si es una fiesta y no hay algo que pueda comer, mejor como llegando a la casa... Persona con ERC. Ent.06

...lo que yo hago es tratar de comer antes y, cuando no como antes, trato de comer arroz o me lleno con tortilla, pero si hay ensalada pues como más ensalada o tostadas y trato de llevar mi botella de agua... Persona con ERC. Ent. 12

\section{Hábitos alimenticios de las personas con ERC de Villa de Reyes}

Los hábitos alimenticios de las personas con ERC son distintos a lo largo de la semana. Eso se debe a que acuden a hemodiálisis entre 2 y 3 veces por semana, lo que implica modificaciones en la cantidad y calidad de su dieta, teniendo en cuenta que es mayor el número de días que ellos no acuden a la sesión de hemodiálisis, se iniciará la descripción por esos días.

En un día común, los informantes realizan de 2 a 3 tiempos de comida. Pocos realizan colaciones, y la cantidad de alimento que se incluye en cada tiempo es muy variable. Los cereales son los alimentos que predominan en su dieta. Estos se comen varias veces al día. La tortilla y la papa, por lo regular, se incluyen en el desayuno, comida (almuerzo) y cena. El consumo de sopas de pasta y bolillo puede darse en los 3 tiempos principales, el arroz se encuentra principalmente en la comida y el pan de caja se utiliza solo en desayunos y cenas. El pan dulce se consume con regularidad en el desayuno o la cena. Es común que se incluyan de 2 a 3 tipos de cereales en un solo tiempo de comida, por lo general son papas, arroz y tortillas.

El consumo de alimentos de origen animal es muy elevado. El huevo y el queso se consumen diariamente y pueden estar presentes en los 3 tiempos de comida principales; la carne de pollo o res se ingieren únicamente en en el almuerzo, siendo mayor el consumo de pollo; la leche se consume poco y por lo general en el desayuno o cena. Una persona con ERC habla sobre su alimentación: 
...como puro pollo, pero a veces nada más que una vez por semana, a lo mucho dos. Lo que es casi al diario son los frijoles..." Persona con ERC. Ent. 08

Del grupo de leguminosas, el frijol es el único que se consume diariamente, puede encontrarse en los 3 tiempos de comida y generalmente se combina con carne, sopa y nopales. Las habas o lentejas se consumen con menor frecuencia, sobre todo en temporadas en que su venta se incrementa, como en la semana santa. Solamente una joven con ERC mencionó comer soya.

Las grasas se consumen diariamente. El aceite es el que más se utiliza y está presente en los 3 tiempos de comida. La crema y la mayonesa se incluyen principalmente en el desayuno y ocasionalmente la cena. Solo 2 de los informantes mencionaron consumir aguacate, almendras o nueces, pero las dos últimas se incluyen por lo general en la granola.

En consumo de verduras es bajo. El jitomate, la lechuga, la cebolla, el chile, la zanahoria, el chayote y la calabaza son los que más se incluyen en la dieta y se encuentran sobre todo en la comida en forma ensaladas, al vapor o en caldos. Solamente uno de los informantes consume las verduras en cremas. Durante el desayuno, solo están presentes como complemento en tortas, sándwiches, quesadillas y, durante la cena, no se consumen. Una cuidadora describe el consumo de verdura de su hijo con ERC:

...pues verduras, así, que brócoli, la calabacita, chayote casi no come... Cuidador. Ent. 12

El consumo de frutas es muy esporádico por su alto costo, así lo manifiestan la mayoría de los entrevistados. La fruta que más se consume es la manzana. Otras frutas como el melón o el limón se consumen principalmente en agua durante la comida y enteras como colaciones. Los dulces y botanas caseras se consumen poco. El refresco se consume casi diariamente y se prefiere el refresco de sabor al refresco de cola.

El día previo y posterior a la sesión de hemodiálisis la alimentación comienza a variar un poco. En el caso del primero, la mayoría lleva una dieta más libre e incluye todo tipo de alimentos e incluso incrementa las cantidades. El día posterior a la hemodiálisis, algunos informantes mencionaron que comienzan a restringir la cantidad, el tipo de alimentos y bebidas, sobre todo cuando es el último día de sesión en la semana por lo que deberán pasar de 3 a 4 días sin hemodiálisis. De este modo, se "autorregulan" y tratan de evitar los malestares y la retención de líquidos para la próxima sesión. Una persona con ERC describe este hecho:

...por ejemplo, hoy, que no voy a la hemodiálisis, ni mañana, le bajo, le empiezo a las cosas, al líquido, a todo... Persona con ERC. Ent. 11 
La alimentación durante el día de la hemodiálisis suele ser distinta a la de un día común, la principal diferencia entre la comida de un día normal y el día que se asiste a hemodiálisis es que deben realizar comidas fuera de casa. Algunos llevan sus alimentos preparados y otros compran en los lugares cercanos. El número de comidas varía según el horario de las sesiones, aunque por lo general se realizan 3 comidas y una colación.

Algunos informantes mencionaron que omiten el desayuno. Esto se debe a que lo asocian con náuseas y calambres durante la hemodiálisis o simplemente por falta de tiempo. Solo una persona refirió tomar un desayuno completo y una colación en el traslado, el resto opta por ingerir solo una fruta o algún alimento ligero durante el traslado a las unidades, las siguientes citas dan cuenta de estas prácticas:

...al principio, antes de que entrara a la hemodiálisis, si desayunaba, pero ya estando ahí me da asco, ya mejor como hasta que salgo y en la noche ceno bien... Persona con ERC. Ent. 11

...aquí desayuna bien y luego ya vamos allá dando la vuelta pa San Luis y ya va comiendo otra vez. Le llevo taquitos de queso y su fruta...Cuidador. Ent. 10

La comida posterior a la hemodiálisis es un punto importante debido al desgaste que provoca este procedimiento, lo que hace que la persona con ERC salga fatigado y con apetito. Ante este hecho, algunos de los cuidadores acostumbran llevarles lanche, ya sea que lo hayan preparado en casa o lo compren en los establecimientos cercanos a las unidades de hemodiálisis para que ellos puedan comer inmediatamente después de la sesión,

...sale con mucha hambre de la hemodiálisis, entonces ya le tengo su lonche cuando sale, a veces le traigo dos tortas de jamón, con poquito queso, su aguacate, así, porque no quiere otra cosa... Cuidador. Ent. 14

...pues le compro de lo que hay cerca de la clínica 50, a veces voy al centro de abastos [mercado] le compro una torta... Cuidador. Ent. 09

Otros comen en los puestos de comida que se ubican fuera de los hospitales y unidades de hemodiálisis, dentro de la ciudad de San Luis Potosí, cerca de la central o en el trayecto a Villa de Reyes:

... a veces venimos en el camino y ahi en Pardo [pueblo cercano a villa de Reyes] le compro gorditas o unos taquitos de barbacoa. Ahi hay una persona que los hace más o menos limpios, y ya llegamos aquí y cena... Cuidador. Ent. 09

Cabe destacar que algunos informantes evalúan los establecimientos donde comen, sobre todo en cuestiones de higiene, y procuran acudir a aquellos lugares que conocen y aprueban. 
Desafortunadamente, la mayoría de ellos se encuentra alejada de los hospitales y unidades de hemodiálisis, o simplemente no encuentra ninguno que cubra estos requisitos, por lo que la persona que recién sale de sesión permanece en ayuno hasta que llegan a casa en Villa de Reyes. Así lo describieron algunos de ellos:

...él sale de la hemodiálisis y nos vamos a almorzar con una tía, que vende en el mercado ambulante. Yo voy ahí porque yo sé que es mi tía y yo sé cómo cocina, que tiene limpio y a veces cuando no está ella, nos vamos hasta la casa porque a mí no me gusta comer en otro lado... Cuidador. Ent. 13

Como se mencionó anteriormente, el traslado de regreso a sus hogares es largo, sobre todo si no se cuenta con un vehículo propio, y esto da la oportunidad a que las personas con ERC realicen colaciones en el trayecto, que por lo general incluyen opciones poco saludables como papas fritas, galletas, jugos de caja y refresco. Una persona con ERC nos habla sobre ello:

... venimos en la camioneta del DIF, ya llegamos a Villa de Reyes y estamos esperando el taxi ahi donde se paran las flechas [autobús flecha amarilla] y este, pos ahi ya nos tomamos unas sabritas [papas fritas industrializadas], un jugo [industrializado] o un sprite [refresco] bien frio y pos ya llega uno sin hambre... Persona con ERC. Ent. 11

La cena es la única comida que realizan en el hogar. La mayoría de los informantes mencionó que es la comida más abundante del día, aunque en ocasiones se la omite debido a que se ingirió alimentos en el trayecto.

Una de las particularidades de la alimentación en los días de hemodiálisis es el tener que comer fuera de casa. Debido a los largos periodos que deben permanecer en la capital y los tiempos para trasladarse, eso se vuelve una práctica común entre todos los participantes del estudio.

Los establecimientos en los alrededores ofrecen alimentos principalmente a base de cereales como gorditas, tacos, tortas, quesadillas, sopes o sándwiches para el almuerzo o desayuno. En algunos lugares, se ofrecen comidas corridas estas incluyen sopa de pasta o arroz, un guisado con carne y frijoles. En el caso de bebidas, se opta por café o refresco.

La mayoría refiere tratar de evitar comer en estos lugares porque considera que los alimentos no son adecuados o simplemente por costumbre, no obstante todos lo hacen. En estos casos, la selección de alimentos es compleja, ya que no hay establecimientos que se ajusten a sus necesidades, las siguientes citas dan cuenta de ello,

...pues es difícil porque tienes que adaptarte a lo que en un momento dado venden en ese lugar, si vas a un restaurante no cambian el menú para que puedas comerlo... Persona con ERC. Ent. 07 
Ante la falta de opciones, los informantes deben elegir que comer, aunque los alimentos que encuentren no cumplan con las recomendaciones, procurando escoger lo más adecuado, pues el proceso de hemodiálisis los deja con mucha hambre y el ayuno prolongado puede acarrear malestares e implicaciones nutricionales inmediatos y a largo plazo.

\section{Uso de medicina alternativa y tradicional}

La medicina alternativa tiene varias ramas. Durante la investigación, los participantes refirieron hacer uso o recibir recomendaciones sobre herbolaría, suplementos alimenticios, vitaminas y algunos alimentos funcionales para desintoxicar el riñón, eliminar cálculos renales, quitar anemia, evitar o dejar la hemodiálisis e incluso curar su padecimiento. Los cuidadores son quienes tienen más conocimiento al respecto. La información se obtiene de otros cuidadores, familiares, conocidos, en programas de televisión o directamente con comerciantes que se dedican a la venta de estos productos, muchos de ellos cerca de los hospitales o unidades de hemodiálisis.

La recomendación de estos productos siempre va acompañada de experiencias de éxito en las que, según quienes las recomiendan y han utilizado, se han disminuido síntomas, incrementado su función renal, reducido el número de sesiones de hemodiálisis y, como algunos hasta refieren, ha quitado la enfermedad. Así lo compartió una informante:

...conocí una señora que me recomendó el palo azul [hierba]. Ella se lo tomaba y le subió la función renal. El doctor se quedó de a seis [impresionado] porque sí se mejoró, nomás tuvo dos hemodiálisis y se le quitó la enfermedad... Cuidador. Ent. 14

El uso de herbolaria fue el que se mencionó con más frecuencia. Se refirieron al palo azul, a la cola de caballo, a los pelos de elote, a la Jamaica y la chaya, los tres primeros para mejorar el riñón y la última para control de la presión arterial. Estas hierbas son utilizadas como té o masticadas en crudo. La siguientes citas dan cuenta de estas prácticas,

...sí, mi esposa se tomaba el té de palo azul cuando almorzaba, en la comida y ya para irse a dormir... Cuidador. Ent. 03

...me he tomado la chaya para la presión. Ahi tengo el árbol. Nada más voy y le arranco las hojas y les masco. Me como dos o tres hojitas por semana... Persona con ERC Ent. 10

Entre los suplementos que comentaron que se les han recomendado y que han consumido, encontramos vitaminas, omega 3, clorofila, malteadas de Herbalife y agua miel. 
Entre los alimentos funcionales que mencionaron utilizar, se encuentran frutas y verduras como la piña, el limón, la berenjena y el noni. La primera la utilizan como diurético y las demás para desintoxicar los riñones y bajar de peso. En todos los casos, el alimento no se ingiere, solo el agua en la que se pone a hervir o remojar. Esas prácticas se las describe a continuación:

... la cascara de piña y el corazón de piña, todo eso se lo hiervo y me lo tomo porque es diurética..." Cuidador. Ent. 10 ...yo estoy tomando la berenjena en agua con el jugo de dos limones. Te ayuda a eliminar toxinas de la sangre, que es lo que a nosotros [los enfermos] nos friega... Persona con ERC. Ent. 08

Por último, se mencionó el consumo de rata de campo y el aguamiel para disminuir la anemia, uno de los trastornos más frecuentes entre las personas con ERC. A continuación se presentan algunas citas que dan cuenta de esta práctica:

...le hago rata de monte pa que se le quite la anemia. Es la rata de monte preparada en caldillo de jitomate con masa. Me lo dijeron mis papás, porque tiene mucha vitamina... Cuidador. Ent. 14

Además de estas recomendaciones, los informantes mencionaron que los médicos naturistas les han prescrito dietas específicas según su tipo de sangre o a base de medicamentos naturistas. En ese sentido, existe controversia, pues algunos enfermos y cuidadores comentaron que este tipo de dieta, a diferencia de la que otorga el nutriólogo, contiene los nutrientes necesarios para el control de su padecimiento, mientras que otros la consideran inadecuada y deciden no hacerla al identificar contradicciones relacionadas con los alimentos que se pueden o no consumir entre ambas dietas e incluso aquellos que por experiencia propia han dejado de consumir porque les producen malestares. Dos informantes hablan al respecto:

...el médico naturista si me da otro tipo de dietas. Son de medicamentos naturistas, pero con nutrientes, porque acá [con el nutriólogo] pos te dan una dieta, pero a veces no lleva los nutrientes que tú necesitas y acá [con el médico naturista] si... Persona con ERC. Ent. 09

...me dio [el médico naturista] una dieta conforme a tu tipo de sangre, pero yo no la hice, es que estaba muy contradictoria por decir ahi me daban 3, 4 veces carnes rojas, el plátano y la papa lo podía comer siempre. $Y$ acá [con la nutrióloga] me decían "no comas tanta carne", y yo ya sabía que la papa y el plátano me hacían daño por el potasio... Persona con ERC. Ent. 12

El hecho de querer entrar al protocolo de trasplante fue referido como una de las causas para evitar el consumo de hierbas, tés y otros remedios, pues los participantes temen que su consumo altere los resultados de los estudios y afecte esta posibilidad. Una persona con ERC comenta al respecto:

...no las he usado. Ahorita no quiero probar nada de eso porque estoy tratando de entrar al protocolo de trasplante y, si como algo que me altere los resultados del laboratorio, no quiero que todo esto se valla para abajo... Ent. 07 


\section{Discusión}

El presente estudio tuvo como objetivo conocer las prácticas alimentarias de quienes viven con ERC en un municipio de México.

De modo general, ninguno de los informantes sigue la dieta prescrita para el manejo y tratamiento de su enfermedad, siguen solo algunas recomendaciones, entre ellas, disminuir el consumo de algunos alimentos y modificar la forma de preparar alimentos. En la literatura, se encuentran trabajos enfocados en estudiar y valorar la ingesta dietética de los personas con ERC, ya sea en el consumo de calorías, macronutrientes, vitaminas y minerales e incluso aditivos, ${ }^{10-14}$ sin embargo no se han explorado a profundidad las practicas alimentarias de esta población.

Los hallazgos de este trabajo coinciden con algunos reportados por autores que han explorado la experiencia de las personas que viven con ERC, como es el caso de Walker y colaboradores, ${ }^{15}$ en que los informantes reportaron haber disminuido o cesado el uso de la sal, sustituyéndola por sal de mar o de hierbas, así como introducido el uso de hierbas de olor y mantequilla para agregar sabor los platillos. Además, se mencionó el uso de tablas y diarios para mejorar el seguimiento de la dieta, mientras que en nuestro trabajo se reportó el uso de vasos graduados y botellas para adecuar la ingesta de líquidos.

Las prácticas alimentarias durante el día de terapia sustitutiva son un hallazgo relevante. Se ha reportado que una de las mayores dificultades al seguir la dieta renal es la falta de opciones alimentarias adecuadas. ${ }^{16}$ En este estudio, se logró identificar qué es lo que los informantes hacen ante esta situación: la mayoría de ellos consume lo que se tiene a su alcance, que, por lo general, no son productos recomendables, como es el caso de alimentos industrializados y refrescos, por su alto contenido en sodio, grasa, azucares y falta de higiene, principalmente cuando se trata de aquellos que se venden en los puestos de comida cercanos a hospitales y unidades de hemodiálisis.

El uso de tratamientos complementarios, herbolaria y alimentos funcionales como medicina alternativa ha sido reportado anteriormente. Dicha práctica es más frecuente en personas con padecimientos crónicos. Eso se coincide con referidos estudios en el uso de té e infusiones de hierbas con mayor frecuencia. ${ }^{17-19}$ A diferencia de lo que se observó en otros estudios, no todos nuestros informantes compartían una visión positiva sobre su uso, aunque este hecho no los exentó de haberlos utilizado en algún momento.

Respecto a la metodología utilizada en este trabajo, la observación participante dentro de los hogares permitió conocer a fondo las prácticas alimentarias. Este método es de gran utilidad para complementar la información obtenida en recordatorios de 24 horas o diarios alimentos utilizados en los estudios mencionados en párrafos anteriores, sobre todo con relación a la selección de alimentos a la hora de comprar y consumir, preparaciones, aplicación de conocimientos adquiridos y seguimiento de recomendaciones dietéticas. En ese sentido, se logró identificar que 
los participantes no utilizan básculas ni medidas caseras, por lo que no hay un control adecuado en el consumo de alimentos.

Una de las limitaciones de este estudio es el hecho de no haberse aplicado herramientas de uso frecuente para la valoración de ingesta dietética como recordatorios de 24 horas, diarios o cuestionarios de frecuencia de alimentos, que en definitiva habrían fortalecido los hallazgos al corroborar, desde el enfoque cuantitativo, que la dieta de la población evaluada es incongruente con las recomendaciones e incluso riesgosa.

Cabe destacar que la inclusión de cuidadores logró enriquecer los hallazgos, ya que se encuentran involucrados casi en la totalidad de las prácticas relacionadas con la alimentación de los enfermos.

Cuadro 1. Características sociodemográficas de las personas con ERC.

\begin{tabular}{ccccccc}
\hline No. & Sexo & Edad & $\begin{array}{c}\text { Estado } \\
\text { Civil }\end{array}$ & $\begin{array}{c}\text { Seguridad } \\
\text { social }\end{array}$ & $\begin{array}{c}\text { Tiempo con } \\
\text { ERC }\end{array}$ & *TRR \\
\hline 1 & M & 19 & Soltero & No & 4 años & Hemodiálisis \\
2 & M & 26 & Unión libre & No & 2 años & Hemodiálisis \\
3 & M & 23 & Unión libre & No & 2 años & Hemodiálisis \\
4 & F & 31 & Casado & No & 2 años & Hemodiálisis \\
5 & F & 40 & Casado & No & 1 año & No \\
6 & M & 20 & Soltero & No & 6 meses & Hemodiálisis \\
7 & F & 30 & Separado & Sí & 10 años & No \\
8 & M & 50 & Casado & Sí & 4 años & Diálisis peritoneal \\
9 & F & 34 & Casado & Sí & 2 años & Diálisis peritoneal \\
10 & M & 26 & Soltero & Sí & 12 años & Hemodiálisis \\
11 & F & 27 & Casada & Sí & 2 años & No \\
12 & M & 65 & Casado & Sí & 3 años & Hemodiálisis \\
13 & M & 45 & Casado & Sí & 12 años & Hemodiálisis \\
14 & F & 26 & Soltera & No & 4 años & Hemodiálisis \\
15 & F & 28 & Soltera & No & 4 años & Trasplante renal \\
16 & M & 50 & Soltero & Sí & 5 años & Trasplante renal \\
\hline Tera & & & &
\end{tabular}

*Terapia de Remplazo Renal 
Cuadro 2. Características sociodemográficas de los cuidadores.

\begin{tabular}{|c|c|c|c|c|c|}
\hline No. & Sexo & Edad & Edo. Civil & Seguridad social & Ocupación \\
\hline 1 & M & 55 & Casado & No & No \\
\hline 2 & $\mathrm{~F}$ & 34 & Casado & No & Ama de casa \\
\hline 3 & $\mathrm{~F}$ & 60 & Casado & No & Ama de casa \\
\hline 4 & $\mathrm{~F}$ & 62 & Casado & No & Ama de casa \\
\hline 5 & $\mathrm{~F}$ & 24 & Soltero & No & Comerciante \\
\hline 6 & $\mathrm{~F}$ & 23 & Unión libre & No & Comerciante \\
\hline 7 & $\mathrm{~F}$ & 46 & Viudo & No & No \\
\hline 8 & $\mathrm{~F}$ & 18 & Unión libre & No & No \\
\hline 9 & $\mathrm{~F}$ & 47 & Casado & Sí & Administrador \\
\hline 10 & M & 50 & Casado & Sí & Abogado \\
\hline 11 & $\mathrm{~F}$ & 50 & Casado & Sí & Ama de casa \\
\hline 12 & M & 45 & Casado & Sí & Pensionado \\
\hline 13 & M & 57 & Casado & Sí & No \\
\hline 14 & $\mathrm{~F}$ & 47 & Casado & Sí & No \\
\hline 15 & $\mathrm{~F}$ & 60 & Casado & Sí & No \\
\hline 16 & $\mathrm{~F}$ & 63 & Casado & $\mathrm{Si}$ & No \\
\hline 17 & $\mathrm{~F}$ & 50 & Soltero & Sí & Jubilado \\
\hline 18 & $\mathrm{~F}$ & 52 & Soltero & Sí & Jubilado \\
\hline 19 & $\mathrm{~F}$ & 46 & Casado & Sí & Ama de casa \\
\hline 20 & $\mathrm{~F}$ & 40 & Casado & Sí & Ama de casa \\
\hline 21 & $\mathrm{~F}$ & 43 & Casado & $\mathrm{Si}$ & Ama de casa \\
\hline 22 & $\mathrm{~F}$ & 44 & Casado & Sí & Secretaria \\
\hline
\end{tabular}

\section{Conclusiones}

Es necesario que nutriólogos y otros profesionales de la salud se interesen por las prácticas alimentarias de la población que padece Enfermedad Renal Crónica, ya que no coinciden con las recomendaciones, lo que implica una mayor incidencia de complicaciones y disminuyen la calidad de vida de esas personas. A su vez, se requiere considerar el contexto de pobreza y desigualdades 
sociales en el que subsisten las personas con ERC y sus cuidadores, para lograr identificar en qué grado se relacionan esas prácticas con las dificultades económicas, inseguridad alimentaria, creencias, gustos y costumbres de los participantes. En ese sentido, es indispensable partir del conocimiento de dichas prácticas para realizar una orientación alimentaria más efectiva y establecer líneas de acción e investigación sobre ese tema.

El uso de la metodología cualitativa, principalmente de técnicas etnográficas y la observación participante, se consideran herramientas de gran utilidad para la elaboración de investigaciones relacionadas con el conocimiento de las prácticas alimentarias y nutrición de la población, así mismo se considera valiosa la inclusión de la voz de los cuidadores, ya que pocas veces se ha considerado la perspectiva de los actores al discutir la problemática del régimen dietético de las personas con ERC.

Este estudio intenta describir y aproximarse a las prácticas alimentarias de quienes padecen ERC. Por lo tanto, podemos decir que estos hallazgos son preliminares y pueden servir para abrir el debate en torno al tema.

\section{Damos las gracias}

Agradecemos a las personas que participaron en esta invetsigación, así como a los miembros de la "Asociación Villa de Reyes con ganas de vivir" A.C, por su apoyo en la identificación de las personas que padecen ERC.

\section{Referências}

1. Levey AS, Coresh J. Chronic kidney disease. The Lancet 2012; 379(9811):165-180.

2. Méndez-Durán A, Méndez-Bueno JF, Tapia-Yáñez T, Muñoz Montes A, Aguilar-Sánchez L. Epidemiología de la insuficiencia renal crónica en México. Dial Traspl. 2010; 31(01):7-11.

3. Walker R, James H, Burns A. A qualitative study exploring patients' perspective of adhering to recommended behaviour change in the predialysis stage of kidney disease. Journal of Human Nutrition and Dietetics 2011; 22(4):405-406.

4. Baraz S, Parvardeh S, Mohammadi E, Broumand B. Dietary and fluid compliance: an educational intervention for patients having haemodialysis. J Adv Nurs 2010; 66(1):60-68.

5. Pasticci F, Fantuzzia L, Pegoraro M, McCann M, Bedogni G. Nutritional management of stage 5 chronic kidney disease. Journal of Renal Care 2012; 38(1):50-58.

6. Alvarez-Gayou Jourgenson JL. Como hacer investigación cualitativa: fundamentos y metodología. México DF: Paidos; 2010. 222 p. (Paidos Educador, 169). 
7. Hsieh HF, Shannon SE. Three approaches to qualitative content analysis. Qual Health Res. 2005; 15(9):1277-1288.

8. Mexico. Ley General de Salud de Estado de San Luis Potosi. Periódico Oficial, el jueves 23 de Diciembre de 2004. Disponivel em: http://www.stjslp.gob.mx/transp/cont/marco\%20juridico/ pdf-zip/leyes/LSESLP/LSESLP.pdf

9. Finlandia. Declaración de Helsinki de la Asociación Médica Mundial. Principios éticos para las investigaciones médicas en seres humanos. DoH Oct. 2008. Disponivel em: http://www.wma.net/ es/30publications/10policies/b3/17c_es.pdf

10. Campos SR, Gusmão MHL, Almeida AF, Pereira LJC, Sampaio LR, Medeiros JMB. Estado nutricional e ingestão alimentar de pacientes em diálise peritoneal contínua com e sem hiperparatireoidismo secundário. J Bras Nefrol. 2012; 34(2):170-177.

11. Martins AM, Rodrigues JCD, Santin FGO, Brito FSB, Moreira ASB, Lourenço RA, et al. Food intake assessment of elderly patients on hemodialysis. Journal of Renal Nutrition 2015; 25(3):321-326.

12. Díaz-López A, Bulló M, Basora J, Martínez-González MÁ, Guasch-Ferré M, Estruch R, et al. Crosssectional associations between macronutrient intake and chronic kidney disease in a population at high cardiovascular risk. Clin Nutr. 2013; 32(4):606-612.

13. Cheung CL, Sahni S, Cheung BM, Sing CW, Wong IC. Vitamin K intake and mortality in people with chronic kidney disease from NHANES III. Clin Nutr. 2014; 34(2):235-40.

14. Karalius VP, Shoham DA. Dietary sugar and artificial sweetener intake and chronic kidney disease: a review. Adv Chronic Kidney Dis. 2013; 20(2):157-164.

15. Walker R., James H., Burns A. A qualitative study exploring patients' perspective of adhering to recommended behaviour change in the predialysis stage of kidney disease. Journal of Human Nutrition \& Dietetics [internet] 2011 [consultado 2012 Dic 28]; 22(4): 405-406. Disponible en: http://web. ebscohost.com.creativaplus.uaslp.mx/ehost/pdfviewer/pdfviewer?sid=2294ae9b-8731-4670-b605-cd 833bb9ea50\%40sessionmgr14\&vid=4\&hid=9

16. Willison KD, Williams P, Andrews GJ. Enhancing chronic disease management: A review of key issues and strategies. Complement Ther Clin Pract. 2007; 13:232-39. 4.

17. Al-Windi A. Determinants of complementary alternative medicine (CAM) use. Complement Ther Med. 2004; 12(2-3):99-111.

18. Ortiz Lucas M, Porcar Boix V, Saz Peiró P. Use of complementary and alternative therapies in a chronic kidney failure population in hemodialysis from Saragossa, Spain. Med Clin (Barc). 2011; 136(11):504-507.

19. Nowack R, Ballé C, Birnkammer F, Koch W, Sessler R, Birck R. Complementary and alternative medications consumed by renal patients in southern Germany. J Ren Nutr. 2009; 19(3): 211-219.

Recebido: 16/4/2015

Revisado: 09/10/2015

Aceito: 15/6/2016 